\title{
Administrative Supplements to Research Grants
}

National Cancer Institute

\section{Source}

National Cancer Institute. Administrative Supplements to Research Grants. NCI

Thesaurus. Code C18616.

These provide administrative supplements to existing NIH research grants for the purpose of 1) promote reentry into biomedical and behavioral research careers 2) promote the recruitment of individuals with disabilities into biomedical research careers and 3) attract underrepresented minorities into biomedical and behavioral research. 